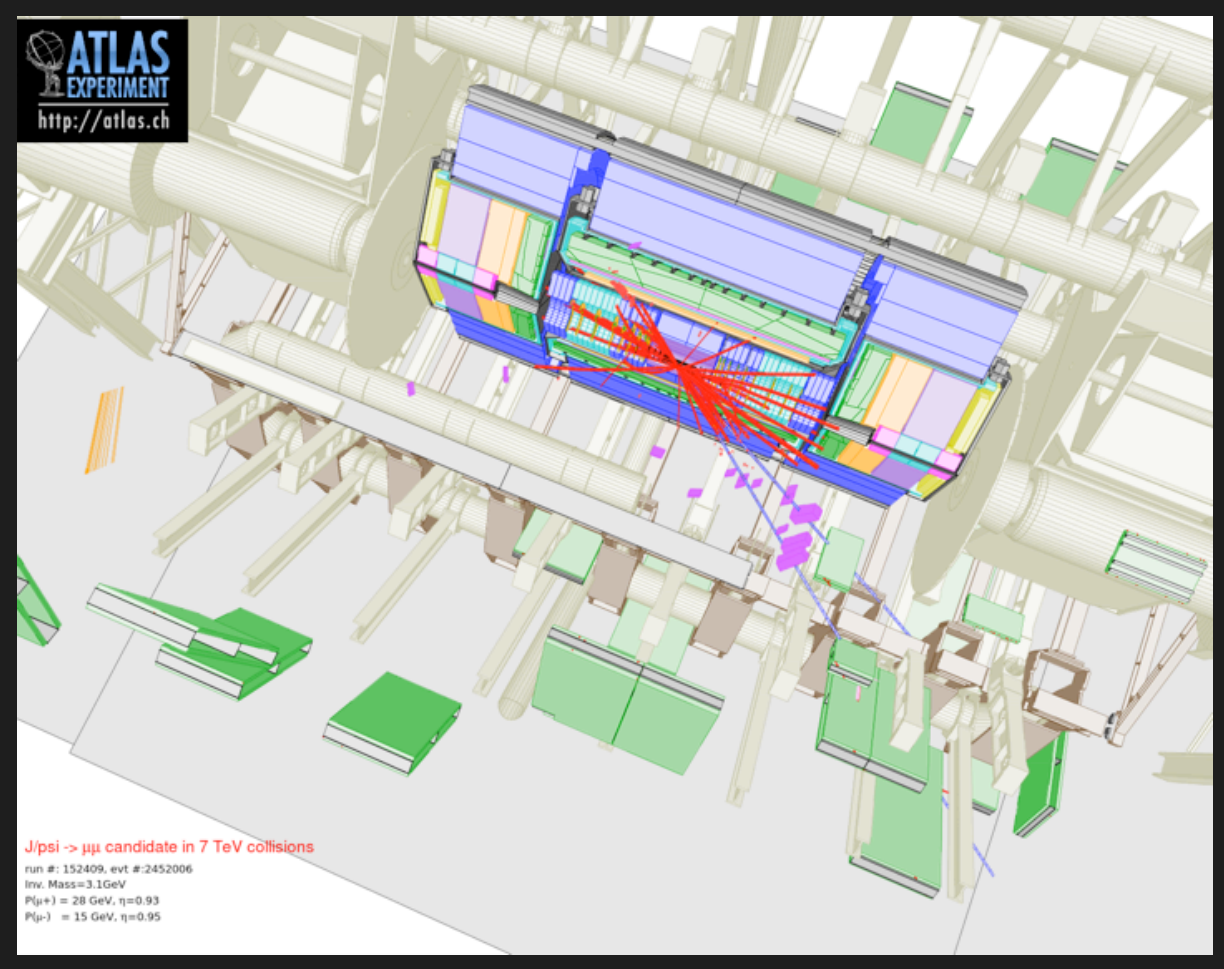

\title{
The charmonium and beauty physics programme in ATLAS
}

\author{
Maria Smizanska \\ Lancaster University, UK
}

The Charmonium and Beauty physics programme in ATLAS, M.Smizanska, BEACH 2010, Perugia 
- ATLAS $J / \Psi$ selection strategy for early beam conditions

- Mass determination, method, results

- Kinematic properties of $\mathrm{J} / \Psi$ with early selections

- First performance results with $\mathrm{J} / \Psi$

- B-physics program

- two examples of early measurements under preparation

- two examples future high sensitivity B-measurements 


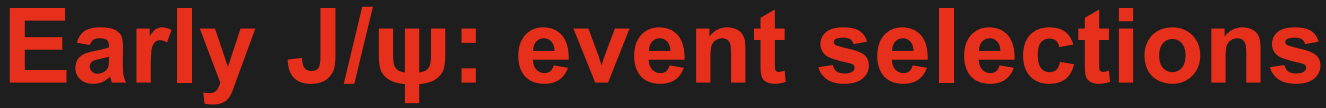

- $\quad p-p$ collision data at $7 \mathrm{TeV}$, taken between March 30th and May 17th 2010

- Integrated luminosity of data used for this study: $6.4 \pm 1.3 \mathrm{nb}^{-1}$

- Strategy:

collect largest possible statistics; determine mass, resolution and $\mathrm{J} / \psi$ properties, understand backgrounds

- Trigger requirements:

- Minimum Bias Trigger Scintillators (MBTS) mounted at each end of the detector in front of the Liquid Argon Endcap-Calorimeter cryostats at $z= \pm 3.56 \mathrm{~m}$. The MBTS trigger - requires at least two hits from either sides of the detector.

- L1 minimum bias trigger was not prescaled for runs with luminosity $<10^{28} \mathrm{~cm}^{-2} \mathrm{~s}^{-1}$.

- A dedicated muon software trigger commissioning chain at the Event Filter level initiated by the MBTS L1 trigger searches for muon track in the entire Muon Spectrometer

- Analysing data in MBTS stream we requested at least one muon must pass the EF muon-commissioning chain with a muon of any $p$ T reconstructed in the Muon System

- To ensure collision events are selected, at least 3 tracks form a primary vertex. 


\section{Types of muons used:}

- Combined muon:

- statistical combination of track parameters and the covariance matrices of Muon System(MS) track and Inner detector (ID) track;

- the tracks with tight matching criteria selected to create a combined muon track traversing the ID and MS

- Tagged muon:

- muon segments matched to ID tracks extrapolated to MS. Reconstructed muon adopts parameters of ID track.

- Pairs of muons with at least one Combined muon were retained

\section{Cosmic ray background:}

- may come from a pair formed by a cosmic muon and a muon from the collision. The probability is very small $\left(<10^{-4}\right)$ from the $900 \mathrm{GeV}$ data analysis

- A cosmic muon mimicking a $\mathrm{J} / \Psi$ decaying back-to-back is excluded - muons detected in the MS can only have momentum higher than $3 \mathrm{GeV}$. 


\section{$\mu \mu$ and $J / \Psi$ selections, cont}

- ID selections, Vertexing:

- $>=1$ hit in the pixels and 6 hits in silicon strip layers

- $\mathrm{pT}>0.5 \mathrm{GeV}$ on each track

- Tracks fitted to a common vertex using vertexing tools based on Kalman filter.

- No constraints on mass or pointing to the primary vertex, and a very high vertex fit $x^{2}$ upper limit is applied $\left(x^{2}<200\right)$.

- Only ID track parameters of muons used for this J/psi study

- Same sign pairs retained for cross-checking.

- Cuts not optimized to reject backgrounds, since the aim of this study is to understand the shape of the low pT combinatorial background 


\section{Early $\mathrm{J} / \Psi$ signal in ATLAS}

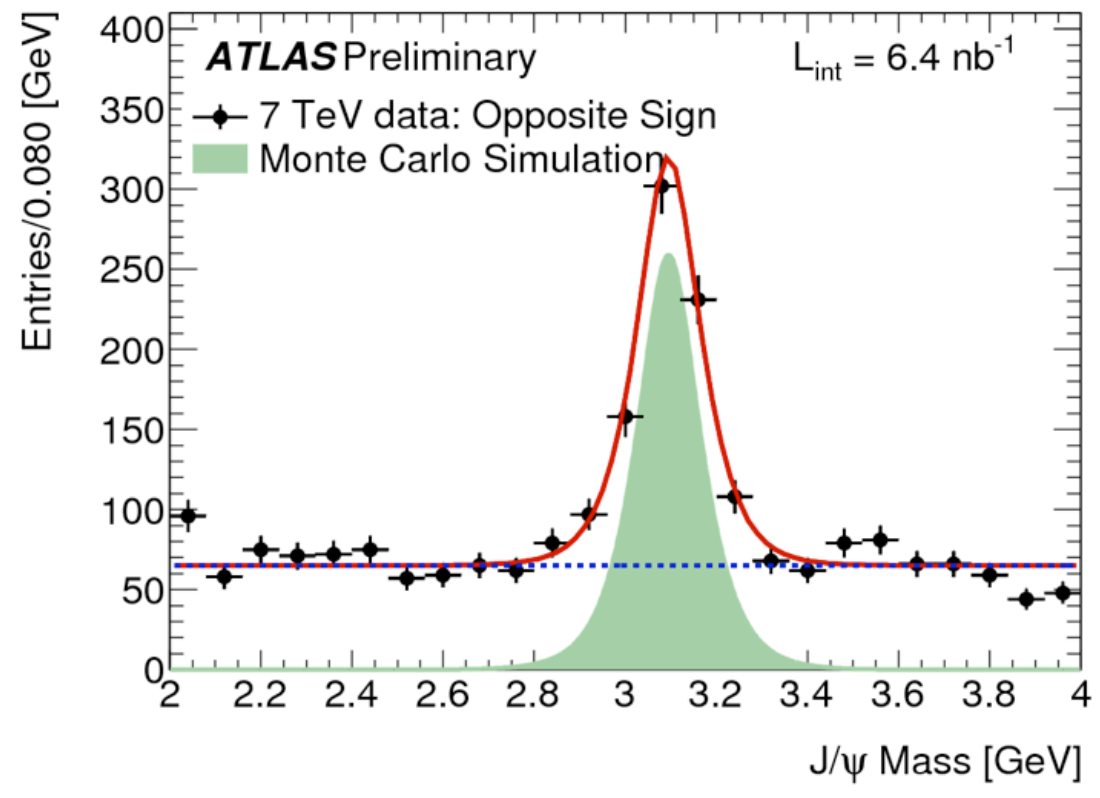

$$
\begin{aligned}
& L=\prod_{i=1}^{N}\left[f_{\text {signal }}\left(m_{\mu \mu}^{i}\right)+f_{\text {bkg }}\left(m_{\mu \mu}^{i}\right)\right] \\
& f_{\text {signal }}\left(m_{\mu \mu}, \delta m_{\mu \mu}\right) \equiv a_{0} \frac{1}{\sqrt{2 \pi} S \delta m_{\mu \mu}} e^{\frac{-\left(m_{\mu \mu}-m_{J} / \psi\right)^{2}}{2\left(S \delta m_{\mu \mu}\right)^{2}}} \\
& f_{\text {bkg }}\left(m_{\mu \mu}\right) \equiv\left(1-a_{0}\right)
\end{aligned}
$$

- $\delta m_{\mu \mu}$ - measured mass uncertainty of each pair of muon tracks

- $\quad$ S - scale factor to cover for unaccounted uncertainties on track parameters (e.g. non-

\begin{tabular}{lccccc}
\hline \hline & & $m_{J / \psi}, \mathrm{GeV}$ & $\sigma_{m}, \mathrm{MeV}$ & $N_{\text {sig }}$ & $N_{b c k}$ \\
\hline all & data & $3.095 \pm 0.004$ & $82 \pm 7$ & $612 \pm 34$ & $332 \pm 9$ \\
& MC & $3.098 \pm 0.001$ & $74 \pm 0.4$ & &
\end{tabular}
gaussian tails)

- The measured mass agrees with PDG within statistical precision of first data

- mass resolution agrees with that expected from MC 


\section{Properties of early J/ in ATLAS}
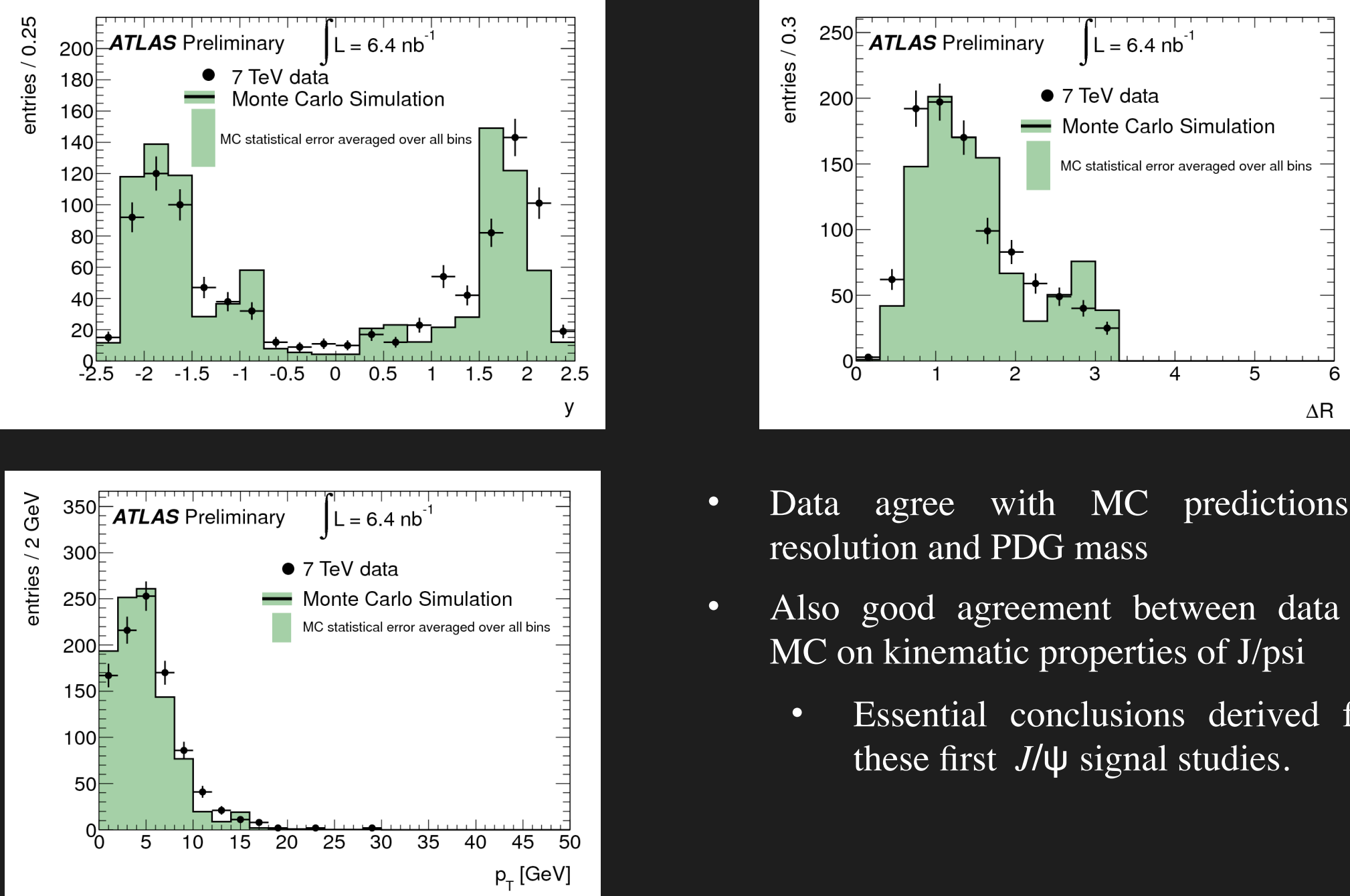

- Data agree with MC predictions of resolution and PDG mass

- Also good agreement between data and $\mathrm{MC}$ on kinematic properties of $\mathrm{J} / \mathrm{psi}$

- Essential conclusions derived from these first $J / \Psi$ signal studies. 


\section{Properties of muons from early $\mathrm{J} / \Psi$}
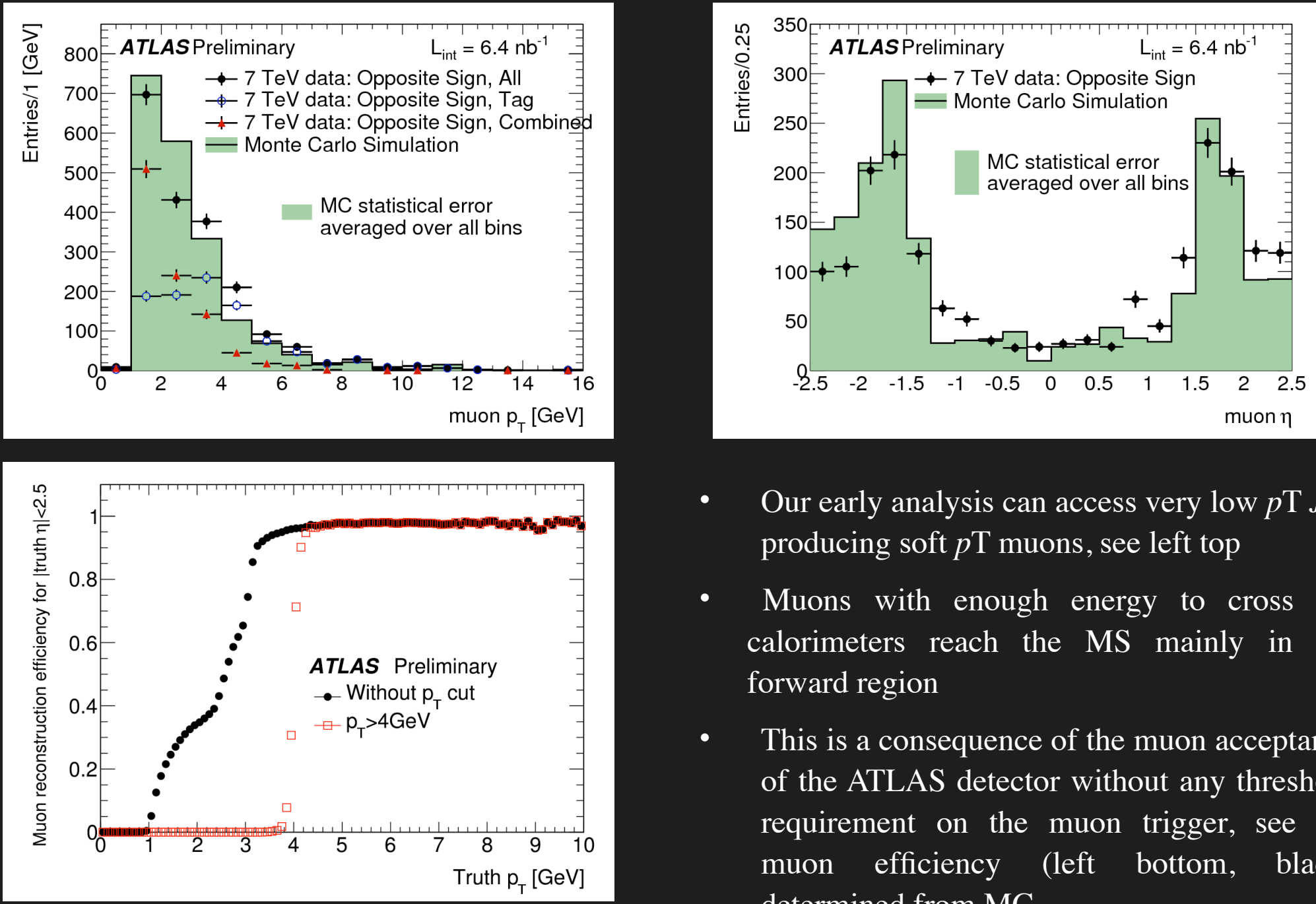

- Our early analysis can access very low $p \mathrm{~T} J / \psi$ producing soft $p$ T muons, see left top

- Muons with enough energy to cross the calorimeters reach the MS mainly in the forward region

- This is a consequence of the muon acceptance of the ATLAS detector without any threshold requirement on the muon trigger, see the muon efficiency (left bottom, black) determined from MC 


\section{Performance of early $\mathrm{J} / \Psi$ in ATLAS}

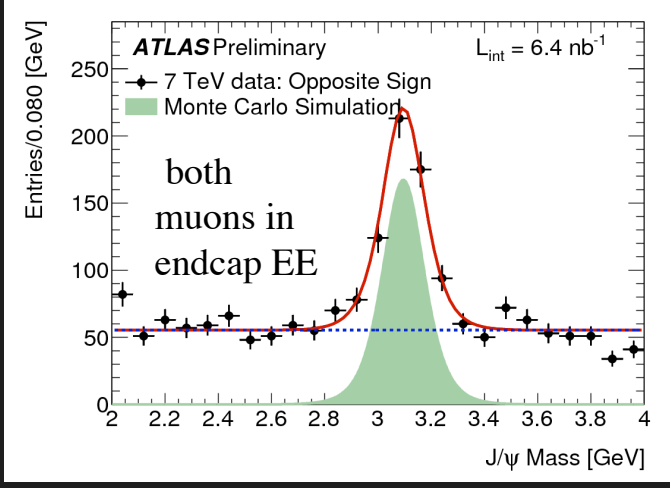

- $\quad J / \Psi$ mass resolution varies with the pseudorapidity of muons accordingly to $\mathrm{MC}$ expectations

- endcap $2.5>|\eta|>1.05$, barrel $|\eta|<1.05$

- no statistically significant mass shifts from the PDG value observed in any of the pseudorapidity regions


\begin{tabular}{llcccc}
\hline \hline & & $m_{J / \psi}, \mathrm{GeV}$ & $\sigma_{m}, \mathrm{MeV}$ & $N_{s i g}$ & $N_{b c k}$ \\
\hline all & data & $3.095 \pm 0.004$ & $82 \pm 7$ & $612 \pm 34$ & $332 \pm 9$ \\
& MC & $3.098 \pm 0.001$ & $74 \pm 0.4$ & & \\
& data n/v & $3.096 \pm 0.004$ & $82 \pm 7$ & $612 \pm 34$ & $351 \pm 10$ \\
\hline BB & data & $3.097 \pm 0.005$ & $36 \pm 6$ & $69 \pm 9$ & $8 \pm 1$ \\
& MC & $3.098 \pm 0.001$ & $37 \pm 0.7$ & & \\
& data n/v & $3.099 \pm 0.005$ & $38 \pm 7$ & $69 \pm 9$ & $8 \pm 1$ \\
\hline EB & data & $3.089 \pm 0.008$ & $66 \pm 12$ & $88 \pm 11$ & $34 \pm 3$ \\
& MC & $3.097 \pm 0.001$ & $53 \pm 0.8$ & & \\
& data n/v & $3.089 \pm 0.009$ & $66 \pm 12$ & $87 \pm 11$ & $36 \pm 3$ \\
\hline EE & data & $3.095 \pm 0.006$ & $88 \pm 9$ & $437 \pm 31$ & $324 \pm 10$ \\
& MC & $3.098 \pm 0.001$ & $82 \pm 0.5$ & & \\
& data n/v & $3.096 \pm 0.006$ & $88 \pm 9$ & $437 \pm 31$ & $344 \pm 10$ \\
\hline \hline
\end{tabular}




\section{Comparison with like sign pairs}

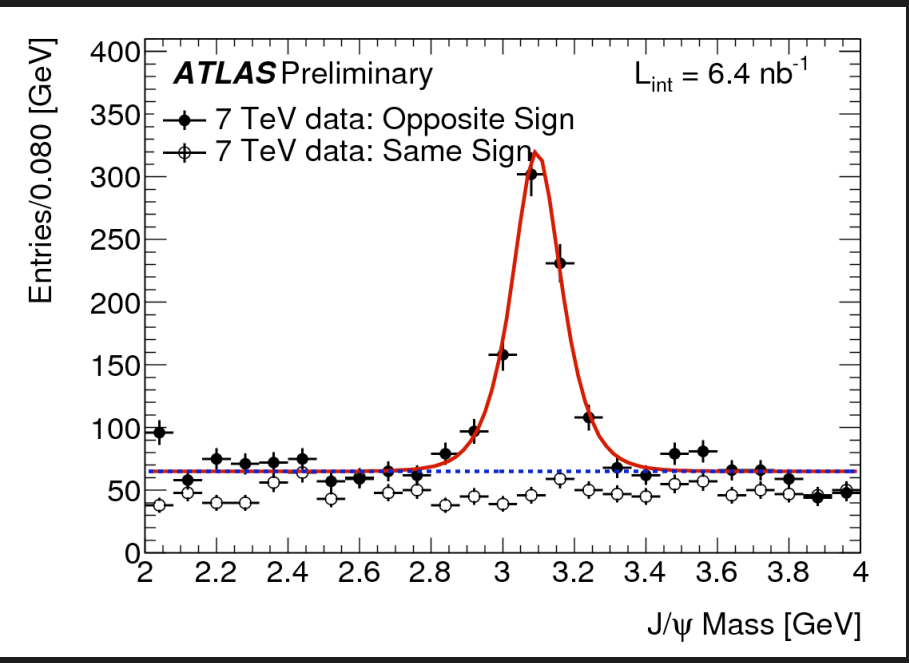

Early di-muon pairs selected at lowest pT have specific features visible when comparing like sign pairs with $\mathrm{J} / \psi$ candidates

- like sign pairs almost match the level of the $J / \psi$ background (unlike pairs) in the side bands

- source of both dominated by muons from K/pi decays

- very little b/c content in tails

Di-muon pairs of opposite sign in the $\mathrm{J} / \Psi$ region have evidently different kinematic properties from the like sign pairs
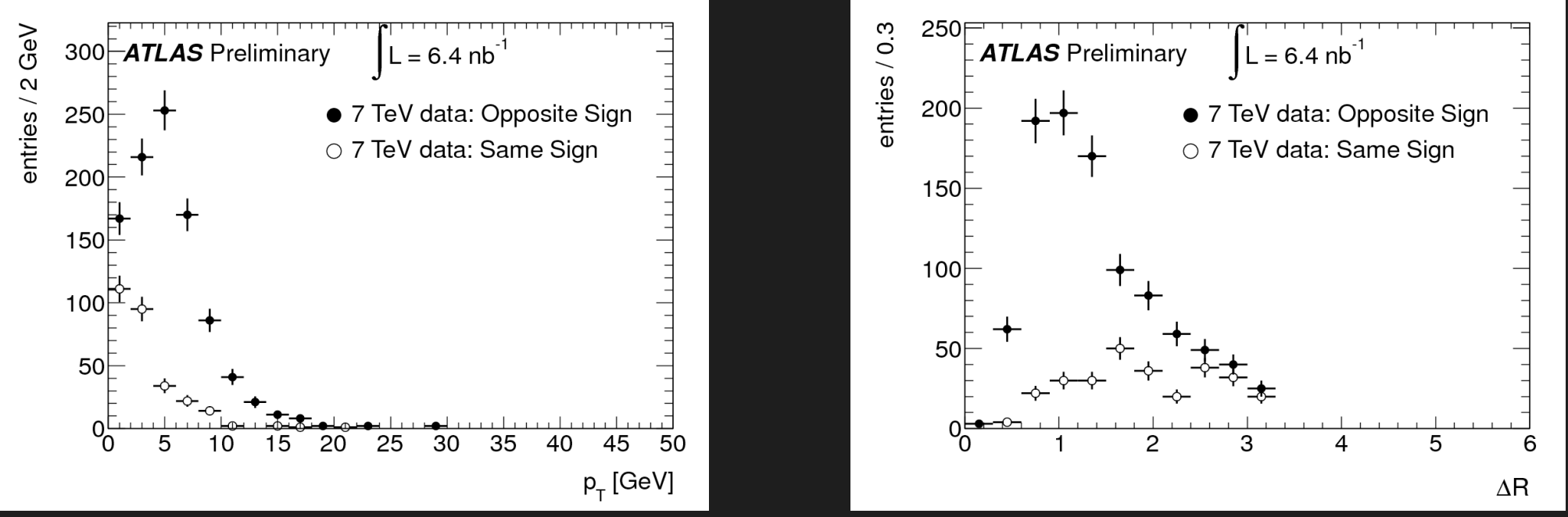


\section{ATLAS B-physics program}

- ATLAS B-physics program is realised in following sub-projects HF quarkonia measurements

$\mathrm{B} \rightarrow \mathrm{J} / \Psi$ (inclusive, exclusive) channels

Rare B-decays $B_{s d} \rightarrow \mu \mu, b \rightarrow s \mu \mu, b \rightarrow d \mu \mu$

Production properties of $\mathrm{B}$ and $\mathrm{D}$-mesons decaying into hadrons

- Each sub-project has tasks/measurements for early, medium and advanced periods

- First measurements, in addition to physics results, serve to improveunderstanding of detector performance to allow later high precision measurements

- Selected examples of MC based studies are given further for the early and for advanced periods

- Complete B-physics program arXiv:0901.0512 ; CERN-OPEN-2008-020, Chapter 11. 


\section{Early measurements with exclusive B}
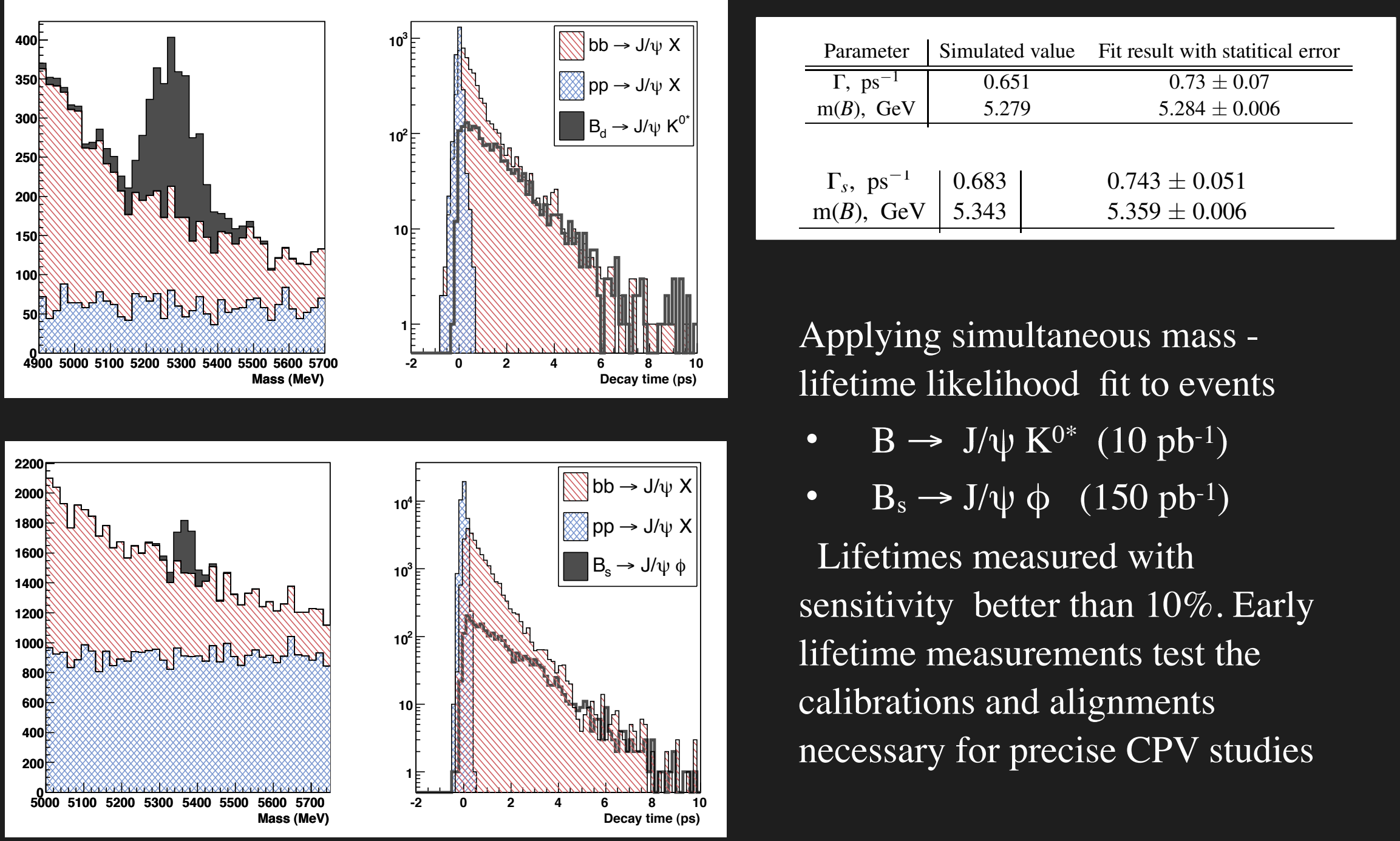

Applying simultaneous mass lifetime likelihood fit to events

- $\quad \mathrm{B} \rightarrow \mathrm{J} / \psi \mathrm{K}^{0^{*}}\left(10 \mathrm{pb}^{-1}\right)$

- $\quad \mathrm{B}_{\mathrm{s}} \rightarrow \mathrm{J} / \psi \phi \quad\left(150 \mathrm{pb}^{-1}\right)$

Lifetimes measured with sensitivity better than 10\%. Early lifetime measurements test the calibrations and alignments necessary for precise CPV studies 


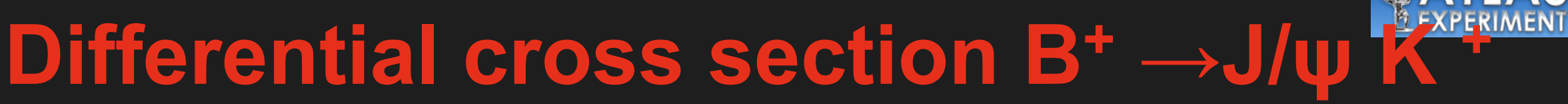
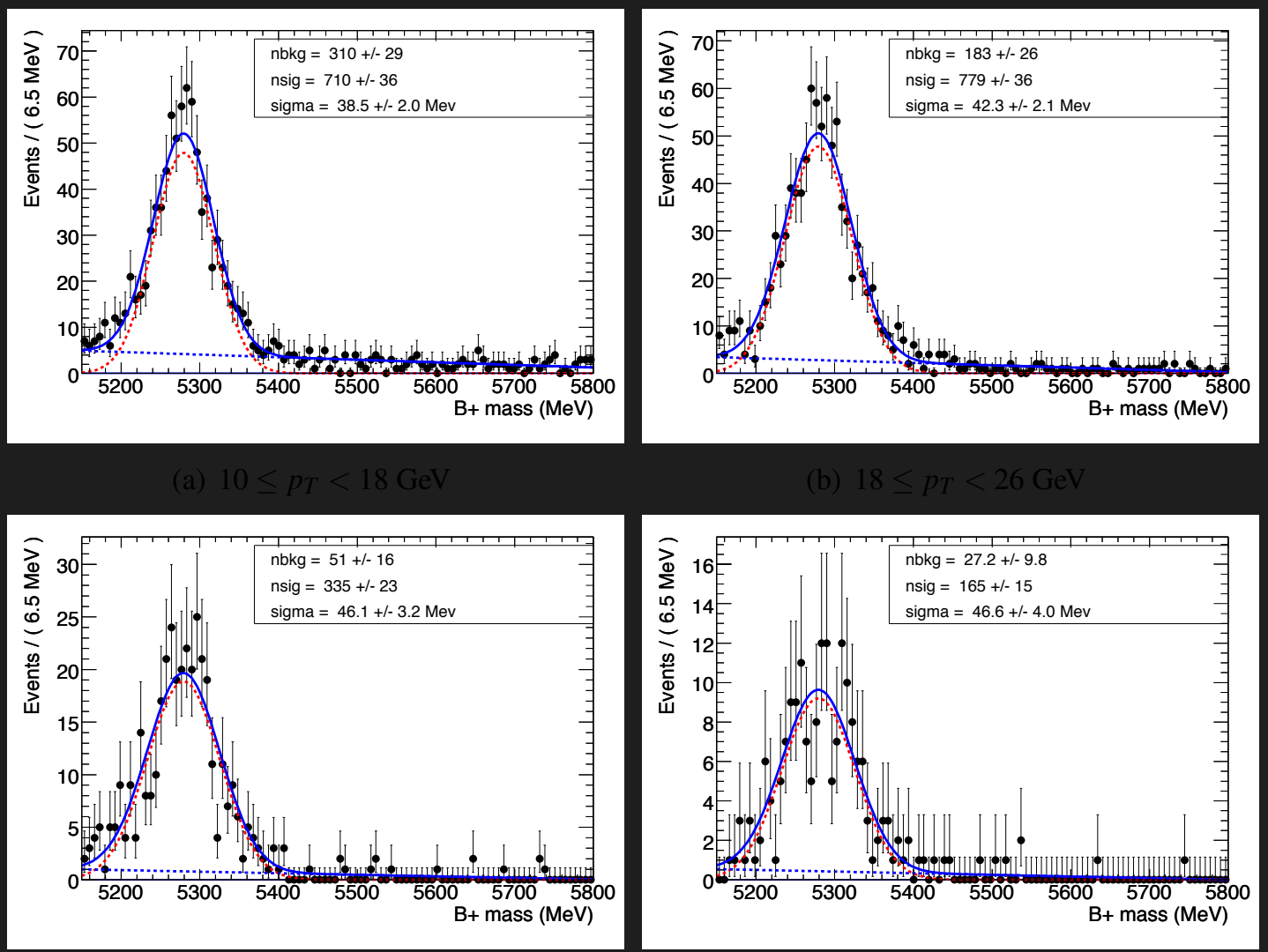

The $\mathrm{B}^{+} \rightarrow \mathrm{J} / \mathrm{KK}^{+}$total and differential production crosssections

- With $10 \mathrm{pb}^{-1}$ the total crosssection can be measured with a statistical precision better than $5 \%$

- The differential cross-section with precision of the order of $10 \%$.

Fit of the $B+$ mass in four $p T$ ranges

\begin{tabular}{|c||c|c|l|l|c|}
\hline$p_{T}$ range $[\mathrm{GeV}]$ & $p_{T} \in[10,18]$ & $p_{T} \in[18,26]$ & $p_{T} \in[26,34]$ & $p_{T} \in[34,42]$ & $p_{T} \in[10$, inf $)$ \\
\hline \hline stat. $+\mathscr{A}[\%]$ & 7.7 & 6.9 & 10.5 & 13.9 & 4.3 \\
\hline total $[\%]$ & 16.1 & 15.8 & 17.6 & 19.8 & 14.8 \\
\hline
\end{tabular}




\section{Production polarization of $\Lambda_{b}$ with 5 iriom}

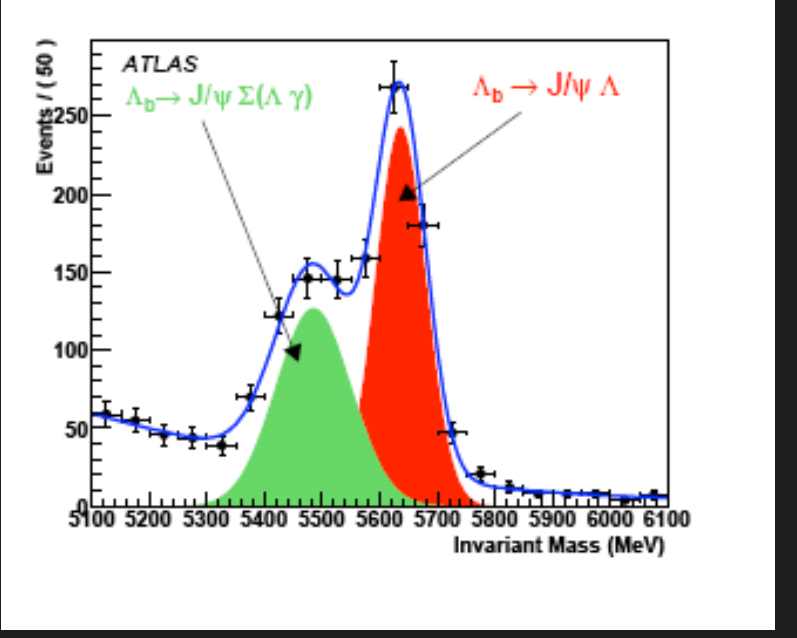

Polarization varies with pseudorapidity thus ATLAS/CMS and LHCb can perform complemetary measurements to map full range.

With $5 \mathrm{fb}^{-1}$ the $\Lambda_{\mathrm{b}}$ polarization in ATLAS can be measured with precision of 0.07

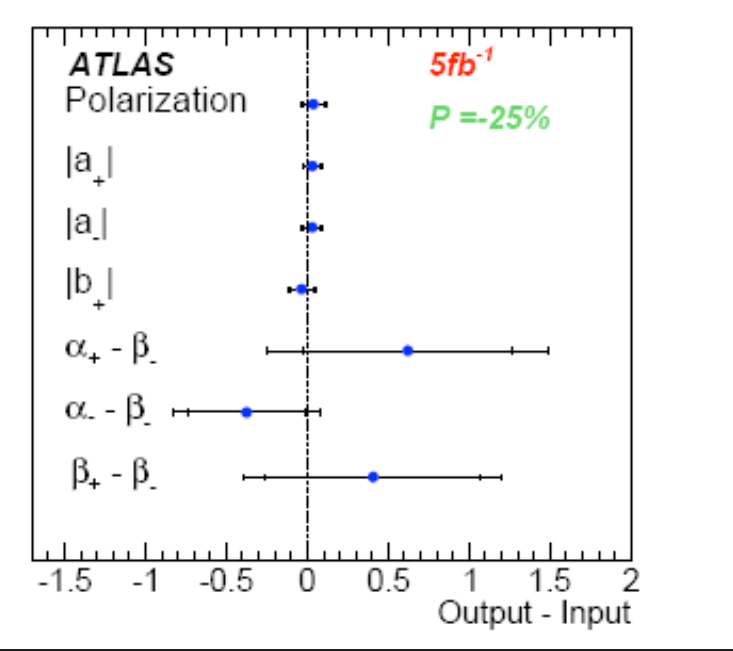

\begin{tabular}{c|c|}
\hline \hline Parameter & $\begin{array}{c}\text { Value } \pm \text { Uncertainty } \\
(\text { Polarization }=-25 \%)\end{array}$ \\
\hline Polarization & $-0.213 \pm 0.069$ \\
$\left|a_{+}\right|$ & $0.461 \pm 0.051$ \\
$\left|a_{-}\right|$ & $0.289 \pm 0.058$ \\
$\left|b_{+}\right|$ & $0.259 \pm 0.071$ \\
$\alpha_{+}-\beta_{-}$ & $-0.991 \pm 0.640$ \\
$\alpha_{-}-\beta_{-}$ & $0.856 \pm 0.364$ \\
$\beta_{+}-\beta_{-}$ & $-1.442 \pm 0.666$
\end{tabular}




\section{ATLAS potential for $B \rightarrow \mu \mu$}

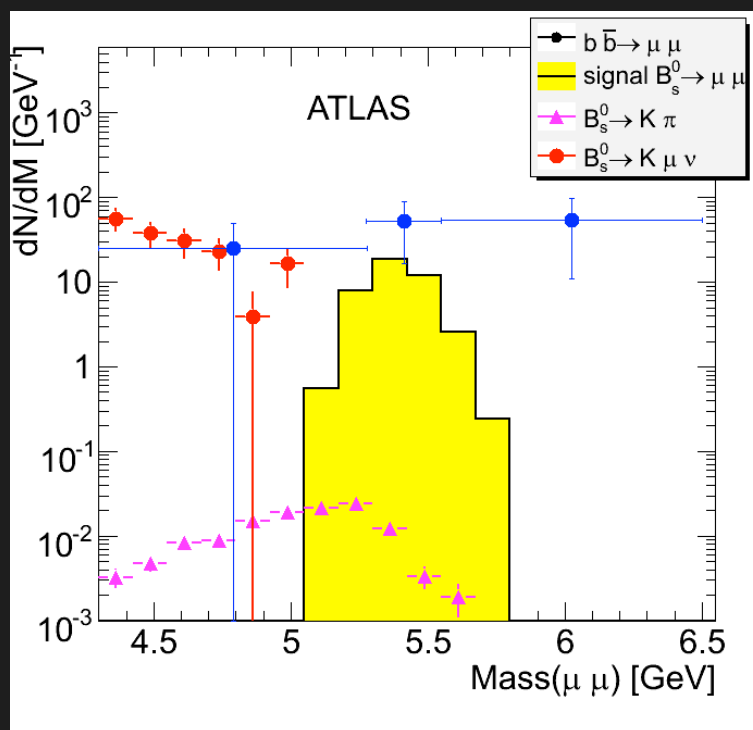

$B_{s} \rightarrow \mu \mu$ signal and backgrounds after applying all selection cuts - relevant at $>10^{33} \mathrm{~cm}^{-2} \mathrm{~s}^{-1}$

\begin{tabular}{|c|c|c|c|}
\hline \hline Selection cut & $B_{s}^{0} \rightarrow \mu^{+} \mu^{-}$efficiency & \multicolumn{2}{|c|}{$b \bar{b} \rightarrow \mu^{+} \mu^{-} X$ efficiency } \\
\hline \hline$I_{\mu \mu}>0.9$ & 0.24 & \multicolumn{2}{|c|}{$(2.6 \pm 0.3) \cdot 10^{-2}$} \\
\hline$L_{x y}>0.5 \mathrm{~mm}$ & 0.26 & $(1.4 \pm 0.1) \cdot 10^{-2}$ & $(1.0 \pm 0.7) \cdot 10^{-3}$ \\
$\alpha<0.017 \mathrm{rad}$ & 0.23 & $(8.5 \pm 0.2) \cdot 10^{-3}$ & \\
\hline Mass in $[-\sigma, 2 \sigma]$ & 0.76 & \multicolumn{2}{|c|}{0.079} \\
\hline \hline TOTAL & 0.04 & $0.24 \cdot 10^{-6}$ & $(2.0 \pm 1.4) \cdot 10^{-6}$ \\
\hline \hline Events yield & 5.7 & \multicolumn{3}{|c}{} \\
\hline
\end{tabular}

- The ATLAS performance was analysed for first $\quad J / \psi \rightarrow \mu \mu \quad$ Shown that di-muon performance with real data consistent with MC predictions

- The MC simulation of $B_{s} \rightarrow \mu \mu$ potential (left) to test potential with $10 \mathrm{fb}^{-1}$ was done with trigger menus for $>10^{33}$

- both muons required pT>6 GeV.

- Low pT B-physics di-muon triggers will be applied at low instantaneous luminosities of early LHC period to maximize reach for first sensitivity

- $\quad$ At $\sim 10^{34}$ dedicated triggers prepared to use full ATLAS potential for $B_{s} \rightarrow \mu \mu$

- $\quad B_{s} \rightarrow \mu \mu B_{d} \rightarrow \mu \mu$ in physics program for ATLAS upgrade 
- Early $\mathrm{J} / \psi$ data taken with minimum bias trigger show excellent agreement with expected performance

- Reproducing $\mathrm{J} / \psi$ PDG mass in all pseudorapidity regions - confirms that pT scale understood at low pT range

- $\mathrm{J} / \psi$ mass resolution over entire pseudorapidity regions of detector - consistent with MC.

- B-physics program prepared for both early and advanced periods.

- ATLAS will significantly contribute to $B \rightarrow \mu \mu$ potential as an instantaneous LHC luminosity will be increased to several times $10^{33}$ and to a nominal value $10^{34}$ Rare B decays for detector upgrade being prepared. 